\title{
Cavitation-Assisted Micromixing for Polymeric Nanoparticle Generation ${ }^{\dagger}$
}

\author{
Salvatore Surdo ${ }^{1, *}$, Mike Alexander Geven ${ }^{2}$, Roberto Donno ${ }^{2}$, Alberto Diaspro ${ }^{1}$, Nicola \\ Tirelli ${ }^{2}$ and Martí Duocastella ${ }^{1}$ \\ 1 Nanophysics, Istituto Italiano di Tecnologia, 16163 Genova, Italy; alberto.diaspro@iit.it (A.D.); \\ marti.duocastella@iit.it (M.D.) \\ 2 Polymers and Biomaterials, Istituto Italiano di Tecnologia, 16163 Genova, Italy; \\ mike.geven@iit.it (M.A.G.); roberto.donno@iit.it (R.D.); nicola.tirelli@iit.it (N.T.) \\ * Correspondence: salvatore.surdo@iit.it; Tel.: +39-010-71781 \\ † Presented at the Eurosensors 2018 Conference, Graz, Austria, 9-12 September 2018.
}

Published: 10 December 2018

\begin{abstract}
The high-throughput generation of polymeric nanoparticles (PNPs) with tailored size and narrow size distribution is key for applications as relevant as sensing and nanomedicine. Here we show how cavitation bubbles in a microfluidic channel can induce rapid nanoprecipitation of PNPs with user-selectable control. Specifically, we used two tip-electrodes perpendicular to the flow to induce electrical breakdown of a polymer solution and a miscible non-solvent. As a result, a plasma is formed causing cavitation and rapid mixing of the fluids, yielding nanoprecipitates of polymer. We demonstrated $\mathrm{mL} / \mathrm{min}$ generation of PNPs with a diameter as low as $150 \mathrm{~nm}$ and polydispersity below 0.15 .
\end{abstract}

Keywords: phase-separation; high-throughput; microfluidics; cavitation bubbles; PLGA

\section{Introduction}

Advances in biosensing and nanomedicine demand for technologies capable of high-yield, fully reproducible synthesis of polymer nanoparticles with controlled size and distribution [1,2]. A promising method to achieve this is microfluidics-assisted nanoprecipitation, namely the generation of PNPs based on mixing of parallel streams of a polymer-solution and a miscible non-solvent in a microfluidic channel [3]. In these processes, easily controlled experimental parameters such as flow rates, flow rate ratios or solution composition allow tuning the size and composition of the generated PNPs [3,4]. In this case, though, several process parameters (e.g., the size distribution) strongly depend on how rapidly and uniformly the two fluids are mixed. For example, to prevent the formation of excessively large nanoparticles, the mixing must be faster than the time needed for particles to aggregate and grow; most often, this condition is not met in bulk processes (e.g., stirring or sonication), whereas in microfluidics the mixing timescale can be faster than that of polymeric aggregation, offering unprecedented control on particle uniformity. However, because most micromixing chips cannot afford simultaneously rapid and complete mixing within a small length-scale, they typically come at the expense of a reduced throughput [5].

Herein we propose a novel implementation of nanoprecipitation in a microfluidic system that addresses the challenges of the existing techniques. Based on the use of cavitation bubbles to induce micromixing at an unparalleled spatiotemporal scale [6], our approach is capable of high-throughput ( $\sim \mathrm{mL} / \mathrm{min})$ generation of PNPs with tailored size and narrow size distribution. 
The simplicity, reduced footprint and efficiency of our system can pave the way for the high-throughput and on-demand generation of customizable functional nanoparticles.

\section{Experimental}

Figure 1a shows a scheme of the cavitation-based PNP generator implemented in a micromixer device. The mixer consists of a T-channel $(\sim 200-\mu \mathrm{m}$-wide) with two stainless steel tip-electrodes (diameter of $200 \mu \mathrm{m}$ and tip-radius of $\sim 50 \mu \mathrm{m}$ ) orthogonal to the main channel (Figure 1b). The T-channel was fabricated in polydimethylsiloxane (PDMS) by using soft-lithography [7]. A portable electronic circuit was used as spark generator (Figure 1c). This circuit generates short ( $\mu$ s time scale) high-voltage pulses (up to $6 \mathrm{kV}$ ) using a rechargeable battery. To control the frequency of the spark, from 16 to $160 \mathrm{~Hz}$, the bias voltage of the circuit can be tuned from 1.2 to $3 \mathrm{~V}$ with a power supply.
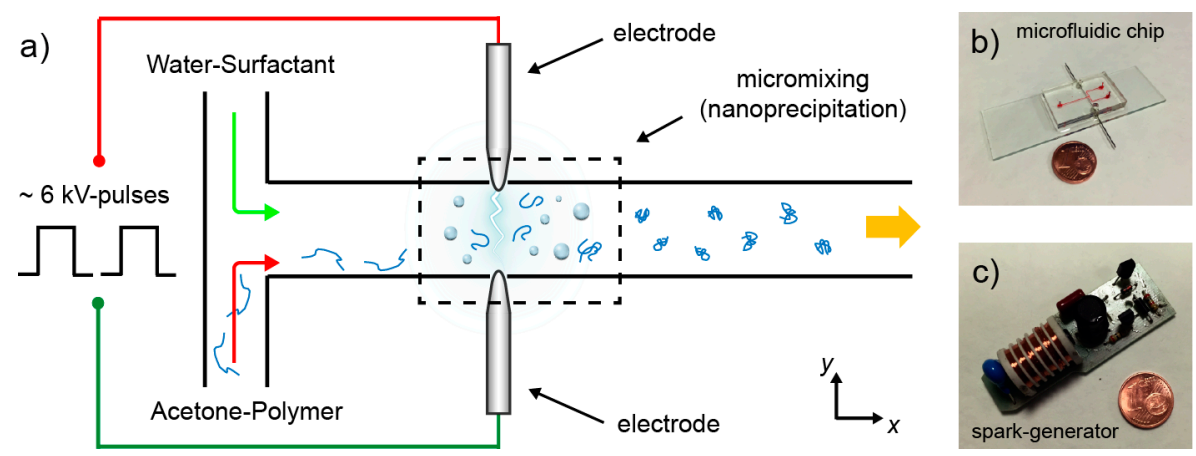

Figure 1. Operating principle and implementation of the cavitation-based PNPs generator. (a) Schematic representation of the micromixing-based nanoprecipitation device. The application of a train of high-voltage pulses between two tip electrodes forms plasma and hence cavitation bubbles. The bubble dynamics, i.e., growth and collapse, rapidly mixes the fluids causing precipitation of the polymers in forms of nanoparticles. (b) Optical image of the microfluidic chip filled with red-colored water for highlighting the $\mathrm{T}$ geometries. (c) Optical image of the spark generator.

The dynamics of the spark-generated cavitation bubble was studied with time-resolved imaging. In this experiment a single high-voltage pulse was used to induce cavitation. Images were captured with an SCMOS camera mounted on an inverted widefield microscope by flashing a high-power white LED for $500 \mathrm{~ns}$. The jitter between the trigger and the LED was $<500 \mathrm{~ns}$, thus enabling a temporal resolution of $\sim 1 \mu \mathrm{s}$. To ensure high reproducibility, 30 images were acquired for each delay time.

Fluorescence microscopy was used for visualizing the mixing process. A fluorescent dye, i.e., nile red, was dissolved in acetone at $20 \mu \mathrm{M}$ and pumped into the main channel through one of the inlets of the T-channel. The second inlet was used for pumping the non-solvent, namely a mixture of water and sodium chloride $(16.7 \mathrm{mM})$. The mixing was evaluated through dilution experiments by recording the fluorescence during the mixing with a widefield microscope at 640 frames per second. In all cases, the excitation wavelength was set at $530 \mathrm{~nm}$ while fluorescence emission was collected in the 590-650 $\mathrm{nm}$ range. The bias voltage during the experiment was 2.25 $\mathrm{V}$, which lead to a spark frequency of $\sim 128 \mathrm{~Hz}$.

The polymer solution was prepared by dissolving poly(D,L-lactide-co-glycolide) (RG502, from Evonik, molecular weight $7-17 \mathrm{kDa})$ in acetone at $0.31 \%(w / v)$ and at room temperature (22 ${ }^{\circ} \mathrm{C}$ ). The non-solvent solution was MilliQ-water with $0.015 \%(w / v)$ of Pluronic F127 (Sigma Aldrich, Milan, Italy) and sodium chloride at $16.7 \mathrm{mM}$.

Dynamic light scattering (Zetasizer Nano ZS, Malvern Instrument, Worcestershire, UK) was used to study the diameter, average and distribution, of the synthesized nanoparticles. To obtain reliable results, several samples $(n=3)$ were analyzed for each flow rate and flow rate ratio couple. 


\section{Results and Discussion}

Initially, we demonstrated that our design is capable of inducing cavitation in a microchannel. To this end, we characterized the electronic circuit used as spark generator. Figure 2 a shows the typical waveform of the high-voltage pulses generated with the circuit. The pulse is a damped sine wave with an amplitude of $6 \mathrm{kV}$ and a period of $25 \mu \mathrm{s}$. As shown in Figure $2 \mathrm{~b}$, the amplitude of this pulse is high enough to induce a visible plasma ( $\mathrm{t} 0 \mu \mathrm{s})$ and, more importantly, a cavitation event. In particular, a cavitation bubble nucleates at the upper electrode, expands towards the middle of channel and finally collapses generating smaller bubbles. Importantly, the particular bubble dynamics enable rapid mixing of two streams of water and acetone solutions, as demonstrated by dilution experiments (Figure 2c). In particular, we found that a degree of mixing of $\sim 70 \%$ is reached in $\sim 10 \mathrm{~ms}$ in a region of interest located only $200 \mu \mathrm{m}$ downstream of the electrodes position. These results confirm the high uniformity and speed of cavitation-micromixing.
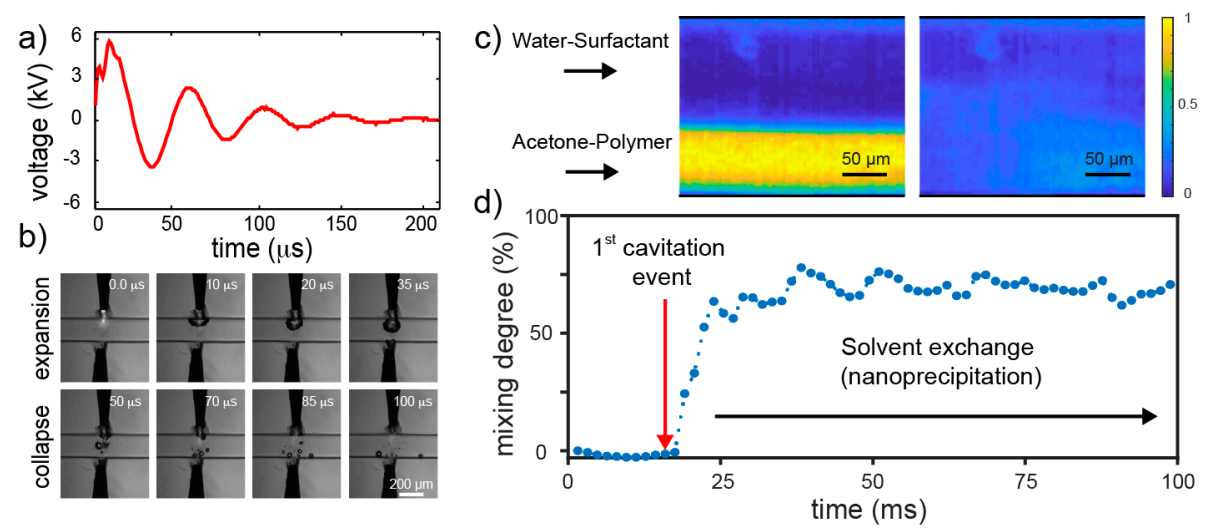

Figure 2. Micromixing with spark-generated cavitation bubbles. (a) Typical voltage pulse produced with the spark generator. (b) Time-resolved images showing the dynamics-formation, expansion, and collapse-of a cavitation bubble generated using a single high-voltage pulse. The flow is from left to right. (c) Surface plots of the dye intensity before and after the first cavitation event and within a region of interest located $200 \mu \mathrm{m}$ downstream from the electrodes position. (d) Degree of mixing vs. time upon the arrival of a sequence of high-voltage pulses with a frequency of $128 \mathrm{~Hz}$.

This high spatiotemporal control allowed us to induce nanoprecipitation of PLGA in water under various experimental conditions. First, we studied the effects of the overall flow rate on the particle size and their distribution. To this end, we induced nanoprecipitation at flow rates ranging from 120 to $960 \mu \mathrm{L} / \mathrm{min}$ and for a flow rate ratio (PLGA-acetone flow/water-surfactant flow) of 0.5 . As shown in Figure $3 a, b$, we found that the cavitation-assisted nanoprecipitation generated NPs with sizes (150-250 nm) significantly smaller than that obtained in the same conditions and without cavitation (210-350 nm). This result clearly highlights the positive effect of the spark-induced cavitation on nanoprecipitation. More importantly, we found a narrow distribution of the nanoparticles size (polydispersity index $<0.15$ ) that further corroborates the high spatiotemporal control of the cavitation-assisted micromixing.

As shown in Figure 3c,d, the flow rate ratio between the acetone and water streams can also be used as a technological parameter to tailor the particle size. In this experiment we induced nanoprecipitation of PLGA at an overall flow rate of $480 \mu \mathrm{L} / \mathrm{min}$ and acetone/water ratio of 0.1 , 0.2 and 0.5 . Interestingly, the size of the precipitated nanoparticles decreases down to $\sim 145 \mathrm{~nm}$ for an acetone/water ratio decreasing from 0.5 to 0.1 . Importantly, the generated NPs are quite uniform with a polydispersity index that decreases to $\sim 0.1$ as soon as the rate ratio decreases from 0.5 to 0.1 . 

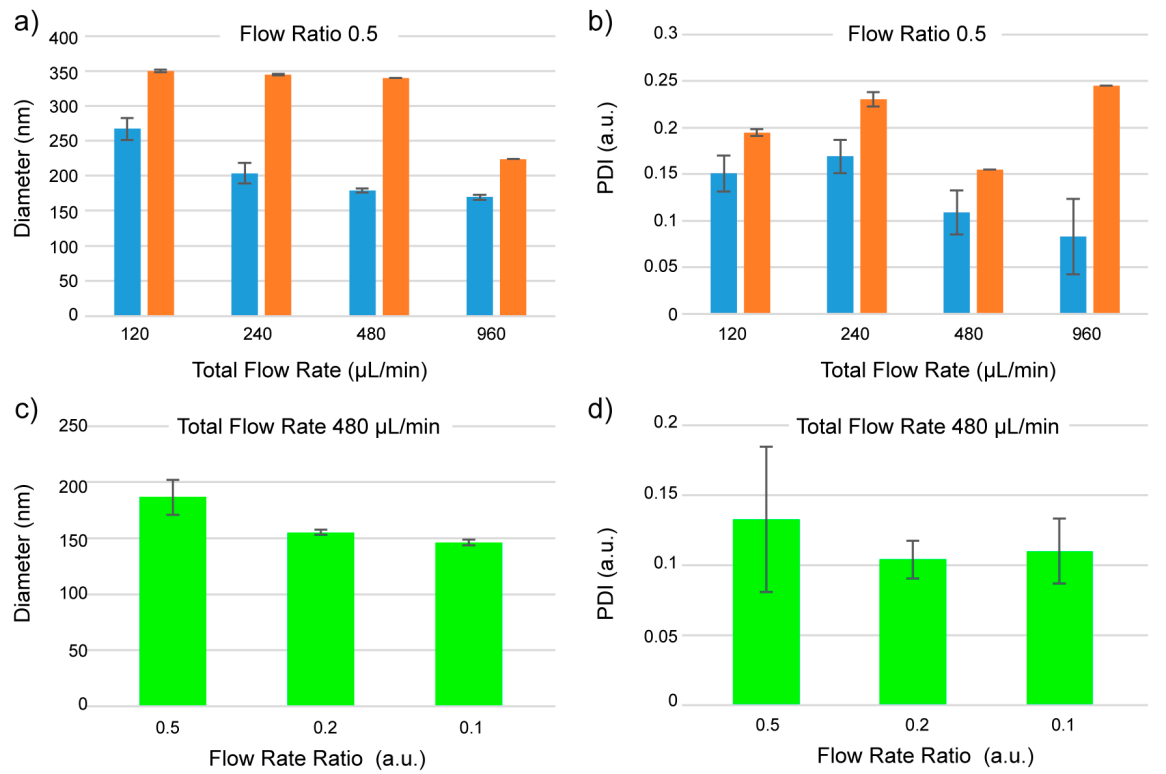

Figure 3. Characterization of the precipitated PNPs. (a) Sizes of the PNPs generated at a flow ratio of 0.5 with (blue) and without (orange) cavitation-assisted nanoprecipitation. The particle size decreases for increasing total flow rates. (b) Polydispersity of the same particles of (a). (c,d) Effects on PNPs both size and distribution of the ratio between the flow rates of the solvent and non-solvent solutions. A reduction of the sizes with decreasing values of the flow rate ratio is evident.

\section{Conclusions and Future Prospective}

The strong perturbation of the spark-generated cavitation bubbles allows rapid and uniform mixing of acetone and aqueous streams in a microchannel. Such high spatiotemporal control of the mixing enables the formation of PNPs that exhibit a narrow size distribution. Importantly, this approach maintains the core advantages of nanoprecipitation method regarding control of the particle size or selection of the chemical composition of the PNPs.

The polydispersity reported here are comparable or lower than those obtained with normally state-of-the-art nanoprecipitation implementations, including batch and micromixer strategies. Even if the obtained throughput is still inferior to those implementations, we believe that the possibility to parallelize the process could significantly improve the performance of our system.

As we continue exploring the pathways for optimization of cavitation technologies, new routes for the on-demand, high-yield production of PNPs with tailored size and composition will continue to emerge, offering new and interesting opportunities for nanomedicine and biosensing.

Conflicts of Interest: The authors declare no conflict of interest.

\section{References}

1. Kamaly, N.; Yameen, B.; Wu, J.; Farokhzad, O.C. Degradable Controlled-Release Polymers and Polymeric Nanoparticles: Mechanisms of Controlling Drug Release. Chem. Rev. 2016, 116, 2602-2663, doi:10.1021/acs.chemrev.5b00346.

2. Miladi, K.; Sfar, S.; Fessi, H.; Elaissari, A. Nanoprecipitation Process: From Particle Preparation to In Vivo Applications. In Polymer Nanoparticles for Nanomedicines, Vauthier C., Ponchel G., Eds.; Springer: Cham, Switzerland, 2016; pp. 17-53.

3. Donno, R.; Gennari, A.; Lallana, E.; De La Rosa, J.M.R.; d'Arcya, R.; Treacher, K.; Hill, K.; Ashford, M.; Tirelli, N. Nanomanufacturing through microfluidic-assisted nanoprecipitation: Advanced analytics and structure-activity relationships. Int. J. Pharm. 2017, 534, 97-107, doi:10.1016/j.ijpharm.2017.10.006.

4. Leung, M.H.M.; Shen, A.Q. Microfluidic Assisted Nanoprecipitation of PLGA Nanoparticles for Curcumin Delivery to Leukemia Jurkat Cells. Langmuir 2018, 34, 3961-3970, doi:10.1021/acs.langmuir.7b04335. 
5. Nguyen, N.-T. Micromixers: Fundamental, Design and Fabrication, 2nd ed.; William Andrew: Amsterdam, The Netherlands, 2012.

6. Surdo, S.; Diaspro A.; Duocastella M. Micromixing with spark-generated cavitation bubbles. Microfluid Nanofluidics 2017, 21, 1-10, doi:10.1007/s10404-017-1917-4.

7. Surdo, S.; Diaspro A.; Duocastella M. Microlens fabrication by replica molding of frozen laser-printed droplets. Appl. Surf. Sci. 2017, 418, 555-558, doi:10.1016/j.apsusc.2016.11.077.

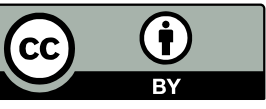

(C) 2018 by the authors. Licensee MDPI, Basel, Switzerland. This article is an open access article distributed under the terms and conditions of the Creative Commons Attribution (CC BY) license (http://creativecommons.org/licenses/by/4.0/). 